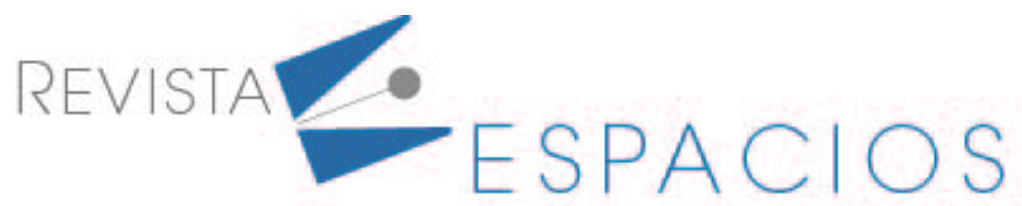

\title{
Comportamiento de los millennials frente al servicio de delivery del sector de restaurantes en tiempos de pandemia
}

\section{Behavior of millennials in front of the delivery service of the restaurant sector in times of pandemic}

SORNOZA, Monica A. ${ }^{1}$

SOTO, Dany F. ${ }^{2}$

Moscoso, Ana $E^{3}$

\section{Resumen}

La presente investigación analiza el comportamiento del consumidor millennial frente al servicio de delivery del sector de restaurantes en tiempos de pandemia. El estudio se desarrolla en la provincia de El Oro, Ecuador; es de carácter descriptivo con metodología cuantitativa, por ello se ejecuta haciendo uso de los métodos de revisión documental y aplicación de encuestas online a una muestra de 384 millennials orenses con edades comprendidas entre los 21 y 41 años. Los principales resultados muestran que, por necesidad y por adaptación, se incrementó demanda del servicio de delivery y que el consumidor millennial prioriza la utilidad del servicio antes que la notoriedad de la marca.

Palabras clave: comportamiento del consumidor, entrega, pandemia

\begin{abstract}
This research analyzes the behavior of the millennial consumer in relation to the delivery service of the restaurant sector in times of pandemic. The study takes place in the province of El Oro, Ecuador; It is descriptive in nature with quantitative methodology, for this reason it is carried out using the methods of document review and application of online surveys to a sample of 384 millennials from Orenses aged between 21 and 41 years. The main results show that, by necessity and adaptation, the demand for the delivery service increases and that the millennial consumer prioritizes the usefulness of the service before the notoriety of the brand.
\end{abstract}

Key words: consumer behavior, delivery, pandemic

\section{Introducción}

A lo largo del tiempo el sector de restaurantes ha sido uno de los sectores que más han aportado a la economía del país, esto debido a que existe una infinidad de negocios, en donde los empresarios han visto una oportunidad para generar fuentes de trabajos e ingresos (Torres, 2013). Una de las estrategias que se han implementado en los últimos años, dentro de este sector, fue la de entrega a domicilio o también denominado "servicio de

\footnotetext{
${ }^{1}$ Estudiante. Facultad de Ciencias Empresariales. Universidad Técnica de Machala. Ecuador. msornoza1@utmachala.edu.ec

${ }^{2}$ Estudiante. Facultad de Ciencias Empresariales. Universidad Técnica de Machala. Ecuador. dsoto3@utmachala.edu.ec

${ }^{3}$ Profesor Titular. Facultad de Ciencias Empresariales. Universidad Técnica de Machala. Ecuador. elmoscoso@utmachala.edu.ec
} 
delivery", la cual consiste en enviar los productos hasta la comodidad de los hogares de sus clientes (UNIVERSO, 2021). Con la pandemia este tipo de servicios ha tenido su auge.

El virus del COVID-19 llegó con muchas restricciones, especialmente el confinamiento que tuvo como consecuencia: el distanciamiento social, Iña limitación de la salida de las personas de sus hogares, el cierre de negocios, la poca afluencia de gente en espacios públicos, la reducción del aforo en locales comerciales, etc. Todos estos cambios tuvieron que enfrentar las empresas y para su supervivencia buscaron y elevaron estrategias para permanecer en el mercado durante la crisis sanitaria (Casco, 2020).

La importancia del delivery radicó en que permitió transportar todo tipo de producto o servicio que las personas demandaban, hasta el lugar donde se encontraban con la seguridad, garantía y sobretodo, con las normas de higiene que ellos buscaban. Para los dueños de restaurantes se trata de un reto el conquistar el mercado de los envíos a domicilio. Sin embargo, los costos del servicio de aplicaciones son altos y no siempre los números dan para asumirlos (COMERCIO, 2021).

Las personas que buscaban este tipo de servicios, principalmente eran personas económicamente activas y con facilidad de uso de la tecnología, la pandemia también incidió en el cambio de conducta o comportamiento de compra de estos consumidores que pasaron de estar habitualmente acostumbrados a comprar de manera física a utilizar por completo aplicaciones móviles para satisfacer todas sus necesidades, entre ellas la de alimentación (Tene, 2020)

Por lo tanto, es innegable el impacto que el COVID-19 ha tenido en el comportamiento del consumidor frente a los servicios de delivery. Estudios recientes han señalado que existen cambios en cuanto al comportamiento de las personas frente al consumo y compra de productos y servicios, por ello, es necesario analizar todos los aspectos que repercuten en su decisión de compra. Así mismo, la información recabada en esta investigación permite tener una visión más clara del segmento millennials, identificando el cambio de hábitos y consumo en el sector restaurantero frente al servicio de entrega de alimentos.

El presente trabajo investigativo tiene por objetivo analizar el comportamiento de los millennials frente al servicio de delivery en tiempos de pandemia, mediante un estudio descriptivo y cuantitativo para que se determine la importancia de este servicio en el sector de restaurantes de la provincia de El Oro.

\subsection{El comportamiento del consumidor}

Los consumidores son seres racionales que buscan satisfacer un deseo o necesidad a través del consumo de un producto o un servicio (Ramírez, Otero, \& Wilson, 2014). Hablar del comportamiento del consumidor es referirse al proceso donde se analiza el por qué y cómo de la compra, es evidenciar el motivo de las decisiones que toman las personas frente a una marca en particular.

Santos (2020), citando a Schiffman (2010), define el comportamiento del consumidor como la conducta que los consumidores exhiben al buscar, comprar, utilizar, evaluar y desechar productos y servicios que ellos esperan que satisfagan sus necesidades. Es decir, que se refiere al estudio de los aspectos que influyen en cada una de las etapas del proceso de compra.

En consecuencia, el estudio del comportamiento del consumidor permite que las empresas definan el perfil de su posible comprador y, con base en esa información, segmentar su mercado y establecer estrategias que permitan anticiparse a sus necesidades para satisfacerlas de mejor manera (Reynolds Mejía, 2013).

Un segmento de mercado que se encuentra económicamente activo en la actualidad, es el segmento millennials o generación $\mathrm{Y}$, se los identifica con este nombre porque han crecido con en el cambio de milenio, son personas 
nacidas entre 1980 y el año 2000, a la presente fecha este grupo de personas están en un rango de edad entre los 21 y 41 años. Según Ordaz \& Osorio (2018) es la generación que se encuentra en la etapa de la juventud, con un estilo de vida inmerso en la tecnología, se caracterizan por buscar un valor agregado en los productos que adquieren y, en relación al consumo en restaurantes, es la generación que gusta de salir a comer, beber y socializar; no obstante, se ven atraídos por los servicios de delivery.

\subsection{Proceso de decisión de compra}

La decisión de compra es un proceso racional donde los clientes potenciales y consumidores experimentan distintas sensaciones y atraviesan distintas etapas, con el objetivo de evaluar que servicio o producto será de su preferencia para resolver un problema o satisfacer su necesidad (Pérez, 2018).

Las etapas del proceso de toma de decisiones al comprar engloban aspectos psicológicos, ambientales y de recursos que son atravesadas por los individuos en su rol de consumidores, por ende, buscan fuentes de información que los ayuden a evaluar las alternativas que tienen a disposición dentro de un sector específico. El posicionamiento y el diseño de estrategias para desarrollar un proceso de compra ideal son claves para que la marca, producto o servicio pueda estar entre las alternativas que considera el consumidor (Pérez, 2018).

Al momento de adquirir o comprar un producto la decisión de hacerlo puede variar en función de la situación o circunstancia del cliente en un momento determinado, sin embargo, muchos consumidores compran por impulso, otros acostumbran a indagar lo que van a comprar y otros dudan y se encuentran indecisos de realizar la compra. Entre las dimensiones que pueden incidir significamente en la decisión de compra constan los grupos de referencias, factores sociales, factores psicológicos, búsqueda de información, comportamiento de compra, estratos sociales, entre otros.

En correspondencia con lo planteado por Martínez y López (2016) las fases o etapas del proceso de decisión de compra son: reconocimiento de la necesidad, donde el consumidor gracias a la publicidad identifica productos que no tiene pero que podría necesitar en función de sus deseos o necesidades; búsqueda de información, se cumple previo a comprar y consiste en recopilar información en fuentes internas o externas en relación a un deseo o necesidad del consumidor; evaluación de alternativas antes de la compra, donde el consumidor compara la información recopilada; decisión de compra, el consumidor realiza la elección y compra del producto; consumo, se refiere a hacer uso del producto o servicio; y, la fase de evaluación posterior al consumo, donde el consumidor evalua sus expectativas y la experiencia de consumo del producto o servicio, experimentando satisfacción o la falta de ella.

\subsection{Servicio de delivery}

El servicio de delivery es para las empresas una de las estrategias de canales de distribución que le permite reducir costos, aumentar la eficiencia en el servicio de atención al cliente y facilitar el proceso de compra para el consumidor; básicamente, consiste en establacer mecanismos para la entrega de sus productos o servicios desde su punto de venta hacia sus clientes o consumidores finales. En consecuencia, se deben especificar las condiciones o políticas del producto o servicio, del pago y el tiempo que demora en llegar a la parte final del proceso de entrega (RANDHAWA, BIRARI, \& JOSHI, 2014).

En la actualidad este tipo de servicio se encuentra implementado en varios sectores comerciales, como lo son farmacias, supermercados, tiendas de barrio, pizzerías, entre otros; de igual manera se refleja a nivel de grandes empresas o en servicios que brindan encomiendas con un tiempo límite.

En relación a servicios de delivery de productos terminados, la entrega a domicilio es de vital importancia porque cumple el objetivo de complacer al cliente y un rol clave dentro de la empresa, puesto que la logística logra su 
función de llegar a más personas en menor tiempo y que las mismas sigan demandando el producto y servicio sucesivamente (Marquez, 2015).

Una distribución de productos bien planificada desde el punto de venta hacia el cliente final, puede representar un enorme ahorro, tanto para la empresa como para los clientes que hacen uso del servicio. Lo que permite que una entrega se vuelva oportuna es relizarla en tiempo reducido, aumentando la producción y la eficiencia de la organización, obteniendo como beneficio la reducción de costos en el servicio (entrega a domicilio).

\subsection{Sector restaurantero}

El sector restaurantero oferta el servicio de venta de alimentos y bebidas para consumo prologando o rápido. Las empresas inmersas dentro de este sector se dedican a la preparación de alimentos para luego comercializarlos dentro o fuera de su establecimiento; estos locales comerciales pueden ser cafeterías, restaurantes, carros de comida, fondas, maquinas que expendan alimentos, hosterías, empresas que realizan comida para llevar, cocinas mercantiles, locales de bufetes o banquetes, comida rápida, fabricación de manteca, venta de tamales, venta de churros, cocinas de aire, marisquerías, loncherías, comedor industrial, platos típicos, refresquerías, merenderos, neveras, jugos y licuados, taquerías, pastelerías, panaderías; y en el caso que cuenten con los permisos necesarios o licencias comerciales podrán comerciar bebidas alcohólicas, así como también ofrecer música, permitir el baile en su local, entre otros, es decir todo establecimiento que cuente con su legalidad y que se dediquen a la venta de alimentos que han pasado por un proceso de preparación (Medina, Román, \& Patrón, 2020).

\subsection{El consumidor millennials en pandemia}

Con la llegada del Coronavirus a nivel mundial se implantaron medidas preventivas en cada país y Ecuador no fue la excepción, el gobierno en ese entonces aplicó el periodo de cuarentena, la cual consistía en el distanciamiento y aislamiento social, provocando varios efectos sobre los hábitos de compra del consumidor millennial. Algunos de los hábitos de compra que se presentan en el consumidor millenial según Quezada, Gualán, \& Ávila, (2020) son:

Acaparamiento: La población actuó de una manera histérica y poco razonable, en cuanto a la compra compulsiva de productos de primera necesidad.

Improvisación: Desarrollaron una actitud optimista y creativa en el sentido de hacer que sus actividades diarias sean llevaderas a pesar de la situación negativa que en los primeros diás de la crisis sanitaria se estaba viviendo.

Demanda acumulada: Se originó un cambio negativo en la tendencia de demanda de ciertos bienes y servicios (autómoviles, electrodomésticos, bienes raíces, cines, conciertos, etc), se pospone la compra de estos productos debido a factores económicos y a ciertas restricciones en cuanto a servicios.

Adopción de tecnología: Hubo un incremento de la adopción de tecnología, debido que los consumidores requerian seguir con sus actividades diarias entre ellas educación, trabajo y salud; y por tal motivo optaron en el uso de ciertas aplicaciones digitales para satisfacer sus necesidades y continuar ese nuevo estilo de vida adaptado.

Con esto se evidencia los cambios que se generaron por el escenario de crisis que se vivía en pandemia, inclusive marcándose diferencia en priopridades de consumo, donde ciertos productos como alimento y medicina pasaron a mayor prefencia en los consumidores millennials. 


\section{Metodología}

El presente estudio analiza el comportamiento de consumo de la generación millennials frente al uso de servicios de delivery en el sector de restaurantes de la provincia de EI Oro en tiempos de pandemia por Coronavirus. La investigación es de carácter descriptivo con metodología cuantitativa, por ello se ejecuta haciendo uso de los métodos de revisión documental y aplicación de encuestas online para recopilar información sobre las variables de estudio.

La revisión documental se utilizó para revisar la información existente en artículos, diarios, fuentes y documentos oficiales sobre el comportamiento del consumidor y el servicio de delivery en el contexto de pandemia; mientras que, la encuesta cumple la finalidad de cuantificar la recopilación y el análisis de los datos sobre el comportamiento de los millennials de la provincia del El Oro frente al servicio de delivery o entrega a domicilio.

Para la aplicación de las encuestas se elaboró un cuestionario de 16 preguntas planteadas a partir de la escala propuestas por Jordán, Ballesteros, Guerrero, \& Pérez (2018) en su estudio de determinantes en el comportamiento del consumidor, con moficicaciones para adaptarlas al contexto y objetivo de la invetsigación (Ver cuadro 1).

Cuadro 1

Comportamiento del consumidor

\begin{tabular}{|c|c|}
\hline \multirow{5}{*}{ Perfil del consumidor } & Edad \\
\hline & Género \\
\hline & Estado Civil \\
\hline & Ocupación \\
\hline & Nivel de ingreso \\
\hline Necesidades del consumidor & $\begin{array}{l}\text { ¿Cuáles son sus necesidades asociadas al servicio de delivery en el sector } \\
\text { de restaurantes? }\end{array}$ \\
\hline Expectativas del consumidor & ¿Qué espera recibir del servicio de delivery de un restaurante? \\
\hline Valores del consumidor & $\begin{array}{l}\text { ¿Qué valores espera percibir en el servicio de delivery de un } \\
\text { restaurante? }\end{array}$ \\
\hline Lugares de compra & ¿Cuál es su restaurante de preferencia que ofrezca servicio de delivery? \\
\hline \multirow[b]{2}{*}{ Frecuencia de compra } & ¿Con qué frecuencia utiliza los servicios de delivery de restaurantes? \\
\hline & $\begin{array}{l}\text { ¿Qué días de la semana usted adquiere el servicio de delivery de } \\
\text { restaurantes? }\end{array}$ \\
\hline \multirow{3}{*}{ Decisión de compra } & ¿Quién decide la compra con servicios de delivery en restaurantes? \\
\hline & $\begin{array}{l}\text { ¿Qué importancia tiene el precio en la decisión de compra con servicios } \\
\text { de delivery? }\end{array}$ \\
\hline & $\begin{array}{l}\text { ¿Considera que la publicidad influye en la decisión de compra con } \\
\text { servicios de delivery en restaurantes? }\end{array}$ \\
\hline Motivos de compra & $\begin{array}{l}\text { Al momento de realizar una compra con servicio de delivery, ¿Cúal es su } \\
\text { principal motivación? }\end{array}$ \\
\hline $\begin{array}{l}\text { Canales de comunicación para } \\
\text { venta }\end{array}$ & ¿A través de qué medio adquiere el servicio de delivery? \\
\hline
\end{tabular}

Fuente: Los autores a partir de Jordán, Ballesteros, Guerrero, \& Pérez (2018)

Para Otzen \& Manterola (2017) la representatividad de una muestra permite extraer ideas de una población que son objeto de estudio, por ello la investigación se concentra en la población de los millennials de la provincia de El Oro con edades comprendidas entre 21 y 41 años. Según datos oficiales del INEC 2010 para el año 2020 existe un universo de 222.742 habitantes en la provincia de El Oro que se encuentra en el grupo de edades que son el objeto de estudio; se aplicó la fórmula para calcular el tamaño de la muestra considerando un nivel de confianza de $95 \%$ y un margen de error de $5 \%$, debiéndose encuestar a 384 millennials orenses. 
Cabe destacar que el tipo de muestreo es no probabilístico por conveniencia debido a que este método permite elegir voluntariamente a los sujetos de investigación según su facilidad de acceso a la muestra (Hernández \& Carpio, 2019).

\section{Cuadro 2}

Plan de explotación

\begin{tabular}{|l|l|}
\hline Universo & $\begin{array}{l}\text { Millennials de la provincia de El Oro con edades } \\
\text { comprendidas entre los 21 a 41 años de edad }\end{array}$ \\
\hline Ámbito geográfico & Provincia de El Oro \\
\hline Tamaño de la muestra & 384 millennials orenses \\
\hline Periodo de recolección de información & Agosto, 2021 \\
\hline Muestreo & No probabilístico por conveniencia \\
\hline Técnicas estadísticas & Análisis descriptivo \\
\hline
\end{tabular}

Fuente: Elaboración propia de los autores (2021)

\section{Resultados y discusión}

\subsection{Resultados}

La data cuantitativa evidencia información interesante, al iniciar la investigación se aplicaron 404 encuestas a través de Google Forms a millennials de la Provincia de El Oro, se indagó si realizaron algún proceso de compra a través desde la comodidad de su hogar y 384 encuestados (95\% de encuestas válidas) aceptaron haber comprando productos utilizando el servicio de delivery, entre los productos más demandados se identificaron alimentos con un $82 \%$, medicinas un $37 \%$ de los enuestados y bebidas un $20 \%$.

A partir de estos resultados, con la finalidad de identificar el comportamiento de los consumidores millennials frente a los servicios de delivery del sector de restaurantes, se planteó como pregunta ¿Usted realiza pedidos de comida a domicilio en restaurantes?, obteniendo como resultado que el 82\% (315 encuestados) si han realizado pedidos de comida a domicilio en restaurantes de su preferencia.

\section{Perfil del consumidor}

Los consumidores millennials que demandan servicios de delivery en restaurantes a nivel de la provincia de El Oro, son mujeres (60\%) de entre 21 y 25 años (72\%) de edad, solteras (72\%) que actualmente se encuentran estudiando (35\%) y trabajando (33\%), con un nivel de ingreso entre $\$ 200$ y $\$ 600$ (48\%) (Ver cuadro 3).

\section{Cuadro 3}

Descripción de la muestra

\begin{tabular}{|c|c|c|}
\hline \multicolumn{1}{|c|}{ Variable } & Frecuencia= 315 & Porcentaje \\
\hline Género & 189 & $60 \%$ \\
\hline Femenino & 126 & $40 \%$ \\
\hline Masculino & 227 & $72 \%$ \\
\hline Edad & 50 & $16 \%$ \\
\hline $21-25$ años & 22 & $7 \%$ \\
\hline $26-30$ años & 16 & $5 \%$ \\
\hline $31-35$ años & 45 & $14 \%$ \\
\hline $36-41$ años & 1 & $0.3 \%$ \\
\hline Estado Civil & 3 & $1 \%$ \\
\hline Casado/a &
\end{tabular}




\begin{tabular}{|c|c|c|}
\hline Variable & Frecuencia $=315$ & Porcentaje \\
\hline Soltero/a & 227 & $72 \%$ \\
\hline Unión Libre & 39 & $12.70 \%$ \\
\hline \multicolumn{3}{|l|}{ Ocupación } \\
\hline Estudia & 110 & $35 \%$ \\
\hline Trabaja & 104 & $33 \%$ \\
\hline Estudia y Trabaja & 82 & $26 \%$ \\
\hline No estudia, ni trabaja & 19 & $6 \%$ \\
\hline \multicolumn{3}{|l|}{ Nivel de Ingreso } \\
\hline Menos de $\$ 200$ & 60 & $19 \%$ \\
\hline$\$ 200-\$ 400$ & 82 & $26 \%$ \\
\hline$\$ 401-\$ 600$ & 69 & $22 \%$ \\
\hline$\$ 601-\$ 800$ & 31 & $10 \%$ \\
\hline$\$ 801-\$ 1000$ & 13 & $4 \%$ \\
\hline Más de $\$ 1000$ & 3 & $1 \%$ \\
\hline No generan ningún ingreso & 57 & $18 \%$ \\
\hline
\end{tabular}

Fuente: Elaboración propia de los autores (2021)

\section{Necesidades del consumidor}

Siendo las necesidades los estados de carencia percibidos por el consumidor y los deseos la forma que adquieren sus necesidades, en el contexto de la presente investigación, dentro las necesidades del segmento millennials asociadas a la oferta del sector de restaurantes, se evidencia (Ver gráfico 1) una alta preferencia por el consumo de comidas rápidas $(56 \%)$ y parrilladas $(33 \%)$ con servicio de entrega de a domicilio.

Gráfico 1

Necesidades del consumidor

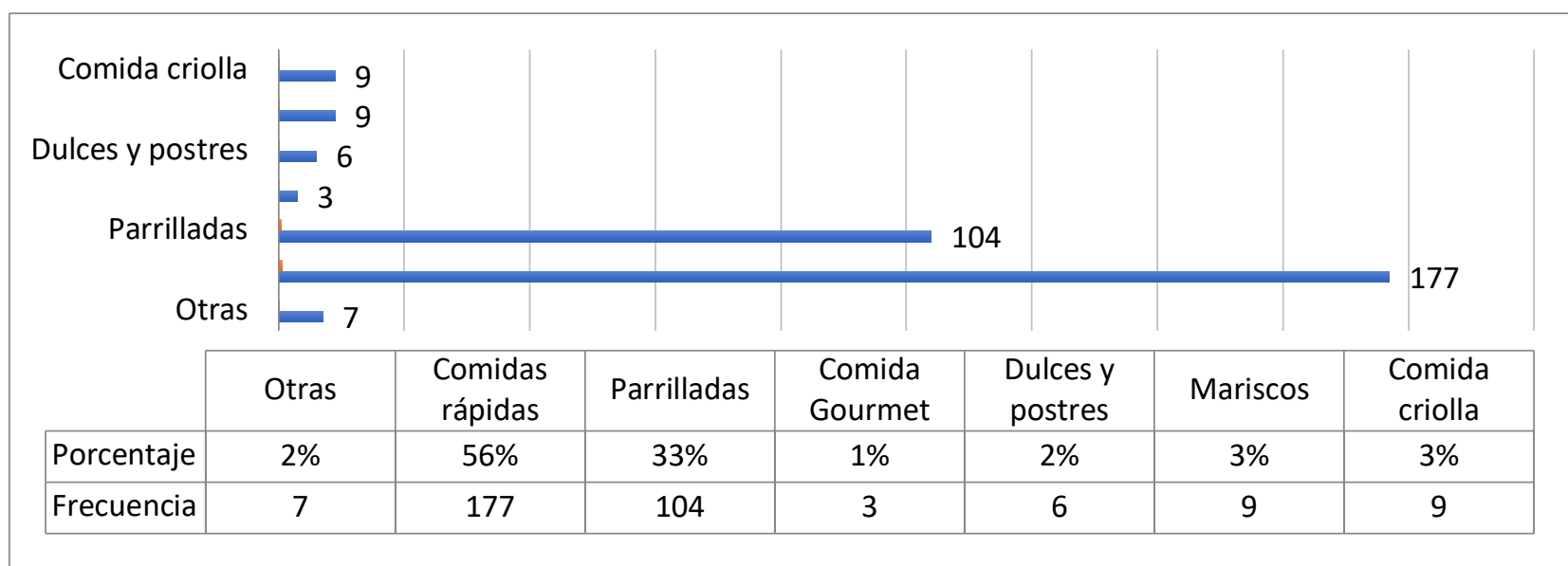

Fuente: Elaboración propia de los autores (2021)

\section{Expectativas del consumidor}

Rápidez (30\%), seguridad (28\%) y salud e higiene (17\%) son las características relevantes que el segmento millennials espera recibir al demandar el servicio de delivery dentro del sector de restaurantes en la provincia de El Oro (Ver gráfico 2). Cabe destacar que por la pandemia salud e higiene se ubica como el tercer atributo del servicio, por encima de la calidad y la garantía que han sido siempre aspectos prioritarios para el consumidor. 
Gráfico 2

Expectativas del consumidor

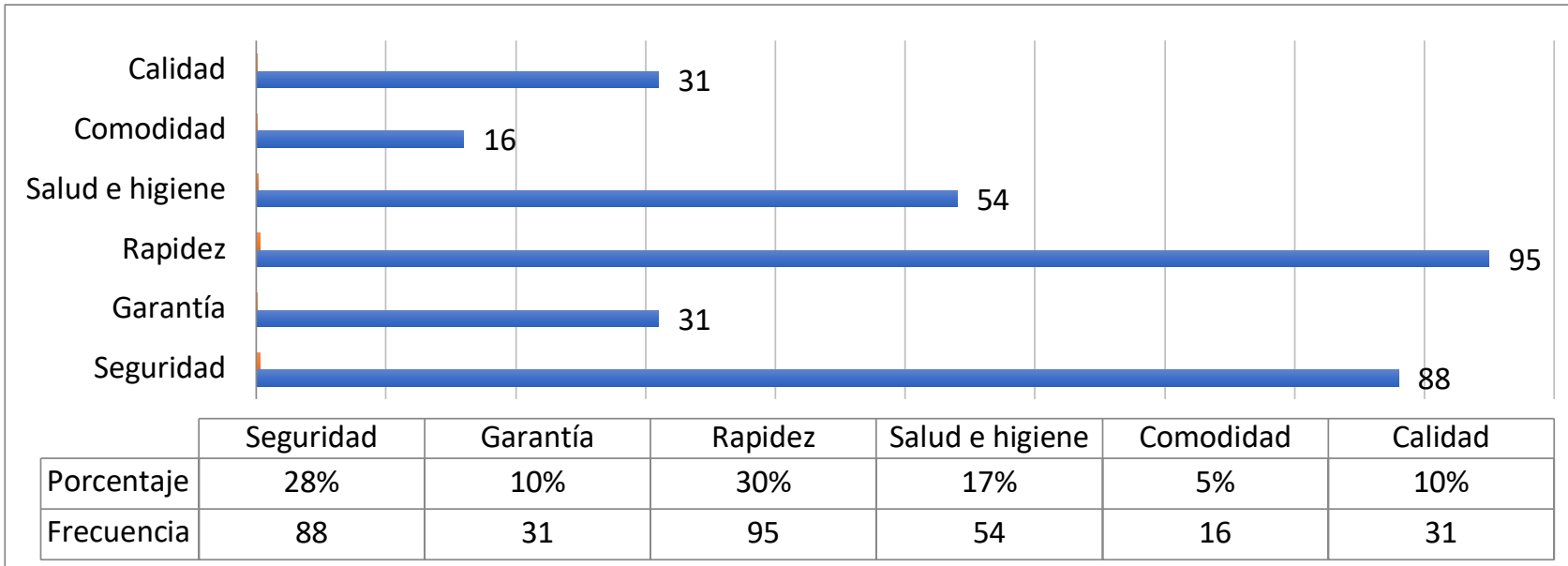

Fuente: Elaboración propia de los autores (2021)

\section{Valores del consumidor}

Los valores de los consumidores tienen una incidencia sobre sus preferencias de compra, por ende, influyen en su conducta y percepción respecto a la marca o su oferta como respuesta a los estímulos que reciben. En consecuencia, en el sector de restaurantes las empresas que cuentan con servicio de entrega domicilio deben considerar que el consumidor millennials valora y espera que el servicio de delivery se caracterice por la puntualidad (88\%), amabilidad (24\%) y el respeto (23\%) (Ver gráfico 3).

Gráfico 3

Valores del consumidor

\begin{tabular}{|c|c|c|c|c|c|}
\hline \multicolumn{6}{|l|}{ Respeto } \\
\hline \multicolumn{6}{|l|}{ Amabilidad } \\
\hline \multicolumn{6}{|l|}{ Generosidad } \\
\hline Honestidad & & & 5 & & \multirow{2}{*}{88} \\
\hline \multirow[t]{2}{*}{ Puntualidad } & & & & & \\
\hline & Puntualidad & Honestidad & Generosidad & Amabilidad & Respeto \\
\hline Porcentaje & $28 \%$ & $17 \%$ & $8 \%$ & $24 \%$ & $23 \%$ \\
\hline Frecuencia & 88 & 54 & 25 & 76 & 72 \\
\hline
\end{tabular}

Fuente: Elaboración propia de los autores (2021)

\section{Comportamiento del consumidor}

A partir de la información recopilada, en este apartado se análizan factores relevantes que influyen y permiten describir el comportamiento del consumidor millennials frente al servicio de delivery en restaurantes de la provincia de El Oro.

Con base en las necesidades del consumidor en relación a la oferta del sector de restaurantes con servicio de delivery, donde se evidencia preferencia por el consumo de comidas rápidas (56\%) y parrilladas (33\%), se indagaron los lugares de compra de estos productos logrando identificar a Chesco Pizzería (44\%) y KFC (26\%) 
como las marcas donde demandan comida rápida mediante servicio de delivery (Ver gráfico 4); en relación a parrilladas, aunque con un porcentaje poco significativo, consta Dollar Grill (9\%). Los porcentajes restantes se distibuyen en un sin número de restaurantes que cuantitativamente no alcanzan un porcentaje significativo entre los resultados (Ver gráfico 1).

Gráfico 4

Lugares de compra del consumidor

\begin{tabular}{|c|c|c|c|c|c|c|c|}
\hline Chesco & & & & & & & 138 \\
\hline KFC & & & & 82 & & & \\
\hline Mc Donalds & 1 & & & & & & \\
\hline Dollar Gril & & 28 & & & & & \\
\hline Parrilladas Grill & (2) & & & & & & \\
\hline La Olla Criola & 1 & & & & & & \\
\hline Otros & & 25 & & & & & \\
\hline & Otros & La Olla Criola & $\begin{array}{c}\text { Parrilladas } \\
\text { Grill }\end{array}$ & Dollar Gril & Mc Donalds & KFC & Chesco \\
\hline Porcentaje & $8 \%$ & $4 \%$ & $5 \%$ & $9 \%$ & $4 \%$ & $26 \%$ & $44 \%$ \\
\hline Frecuencia & 25 & 13 & 16 & 28 & 13 & 82 & 138 \\
\hline
\end{tabular}

Fuente: Elaboración propia de los autores (2021)

La frecuencia de compra es una métrica del comportamiento del consumidor que muestra la cantidad promedio de días o el periodo de tiempo que transcrurre entre una compra y otra. En relación a esta métrica, los resultados permiten determinar que el consumidor millennials demanda los servicios de delivery en restaurantes de forma mensual (43\%), identificándose un incremento en la contración de este servicio de manera semanal (31\%) a razón del contexto actual (Ver gráfico 5).

Gráfico 5

Frecuencia de compra

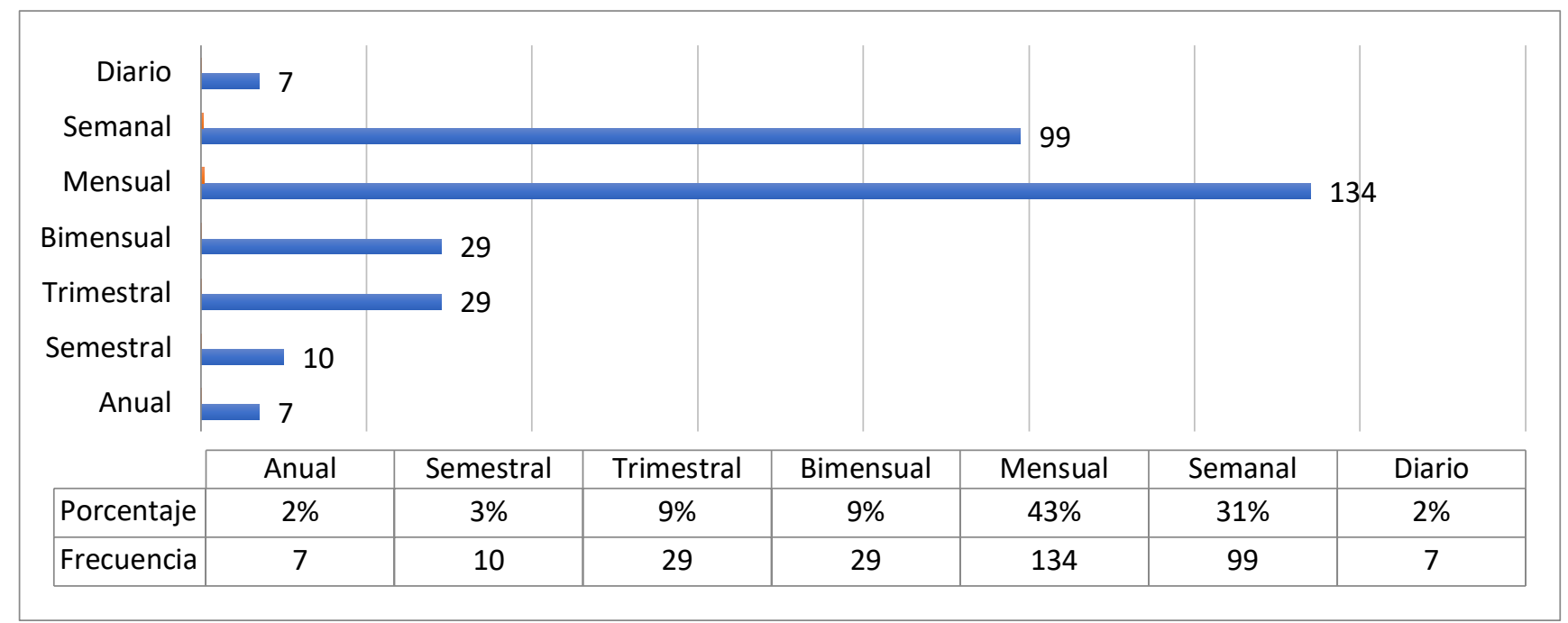

Fuente: Elaboración propia de los autores (2021)

Estudiando el comportamiento de compra en función de los los días de la semana, se determina a nivel general, que el $81 \%$ de los encuestados demandan los servicios de delivery en restaurantes durante el fin de semana 
(viernes 23\%, sábado 35\% y domingo 23\%). Siendo oportuno destacar que el sábado es el día de la semana en el que más pedidos a domicilio se efectúan actualmente, identificándose aquí un cambio en el comportamiento habitual del consumidor, puesto que el sábado era el día de mayor concurrencia en el sector de restaurantes (Ver gráfico 6).

\section{Gráfico 6}

Compra por días de la semana

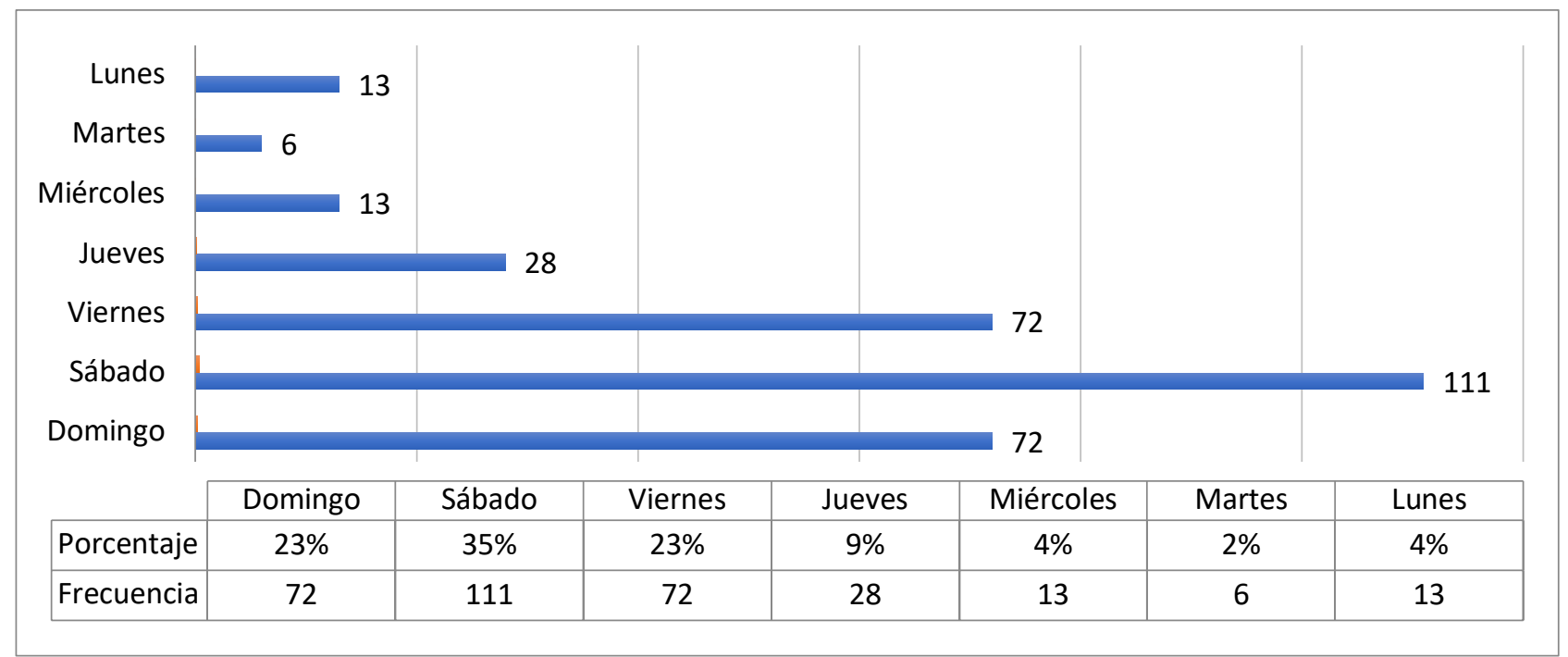

Fuente: Elaboración propia de los autores (2021)

Respecto a la decisión de compra, los resultados evidencian que el consumidor millennials cumple los roles de decisor, comprador y consumidor frente al servicio de delivery de los restaurantes, con base en que el $67 \%$ de los encuestados indican que la decisión de compra se da por iniciativa propia (Ver gráfico 7).

\section{Gráfico 7}

Decisión de compra

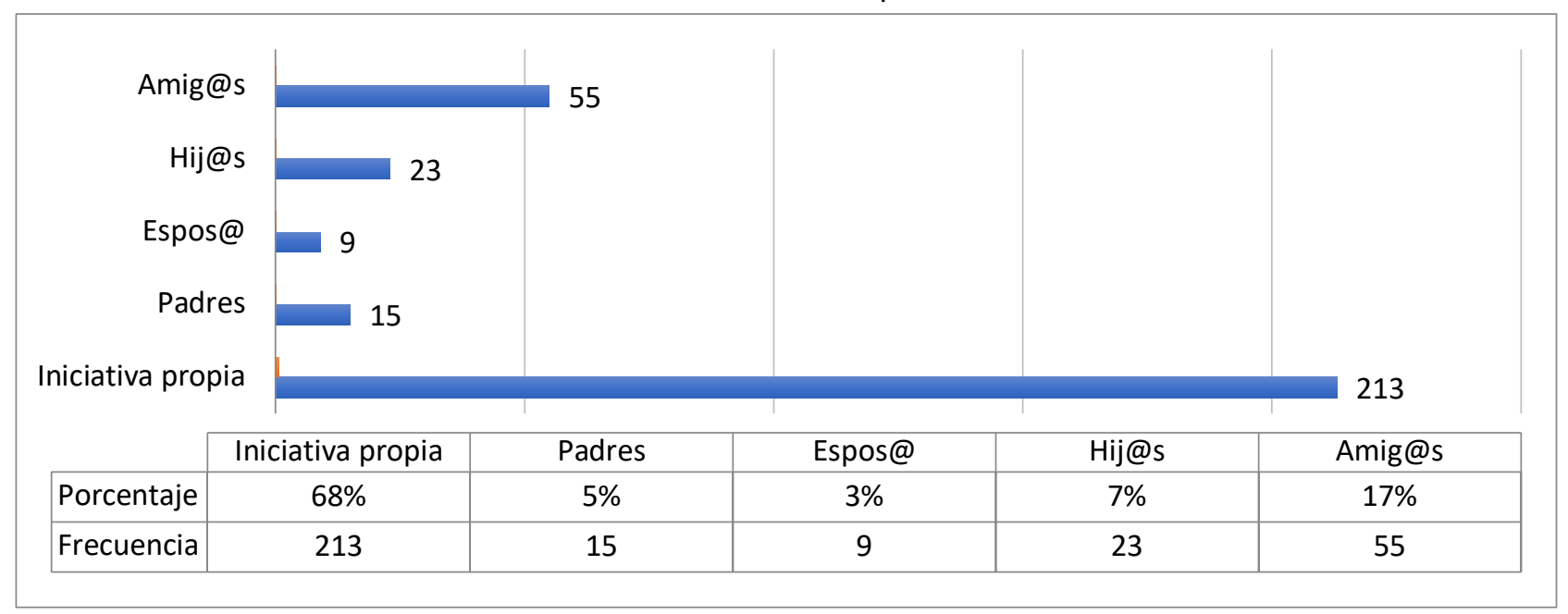

Fuente: Elaboración propia de los autores (2021)

El $81 \%$ de los millennials encuestados consideran que el precio es un factor clave (muy importante $55 \%$ e importante 26\%) al momento de decidir la compra del servicio de delivery en el sector de restaurantes, por ello es fundamental que para los servicios de entrega a domicilio se diseñen políticas que permitan fijar el precio atendiendo el valor percibido por el consumidor (Ver gráfico 8). 
Gráfico 8

Precio en la decisión de compra

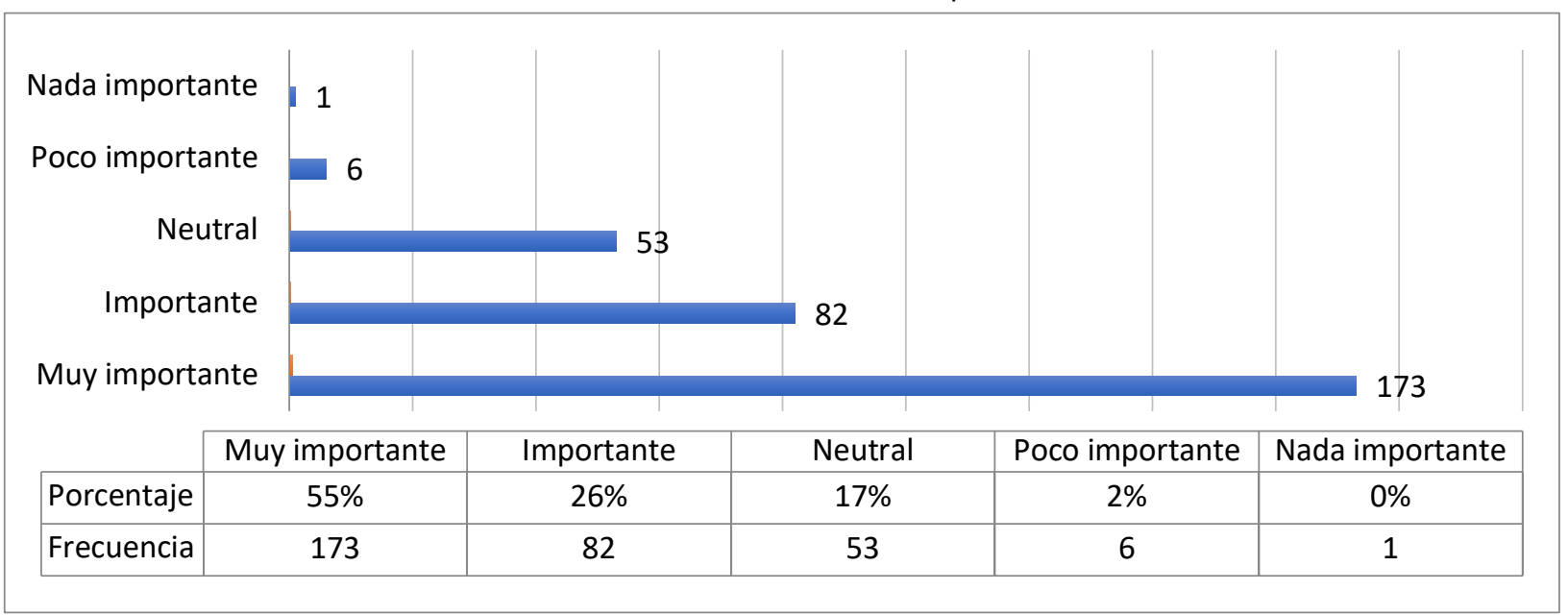

Fuente: Elaboración propia de los autores (2021)

Se determina que la publicidad influye sobre la demanda de los servicios de entrega a domicilio en el sector de restaurantes, a partir de que el $79 \%$ de los encuestados afirman (siempre $48 \%$ y casi siempre $31 \%$ ) que el contenido publicitario incide sobre su elección en el contexto de la investigación (Ver gráfico 9). Esto evidencia la necesidad de invertir en publicidad para promocionar el servicio de delivery, debido a que mejora la capacidad de elección y simplifica los procesos de compra.

Gráfico 9

Publicidad en la decisión de compra

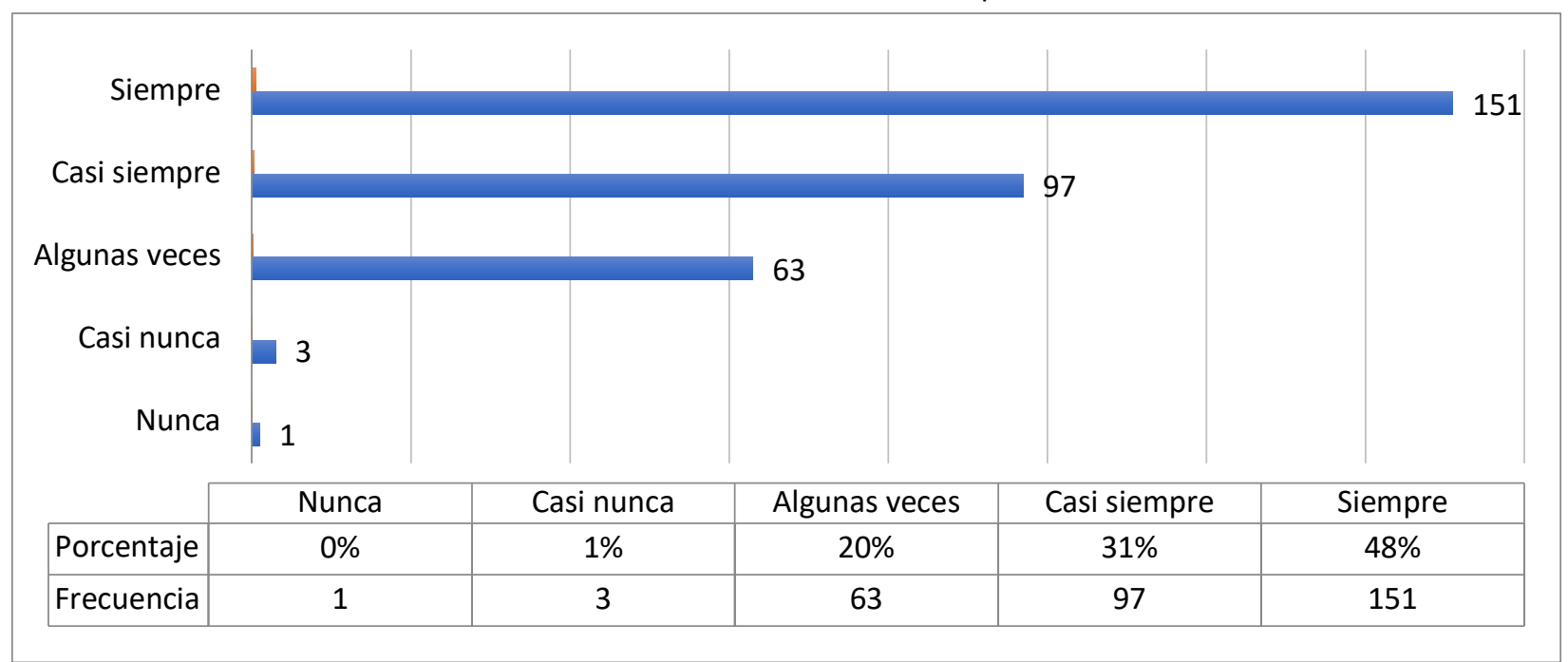

Fuente: Elaboración propia de los autores (2021)

La necesidad (53\%) es el principal motivo de los 315 encuestados que utilizan el servicio de delivery en los restaurantes de su preferencia (Ver gráfico 10), esta respuesta debe estar direcccionada por todos los protocolos y medidas de seguridad que se han implementados por la pendemia. Es oportuno mencionar que un $22 \%$ de los encuestados que demandan este servicio los motiva el impulso o gusto y que un $13 \%$ lo hace por coveniencia. 
Gráfico 10

Motivos de compra

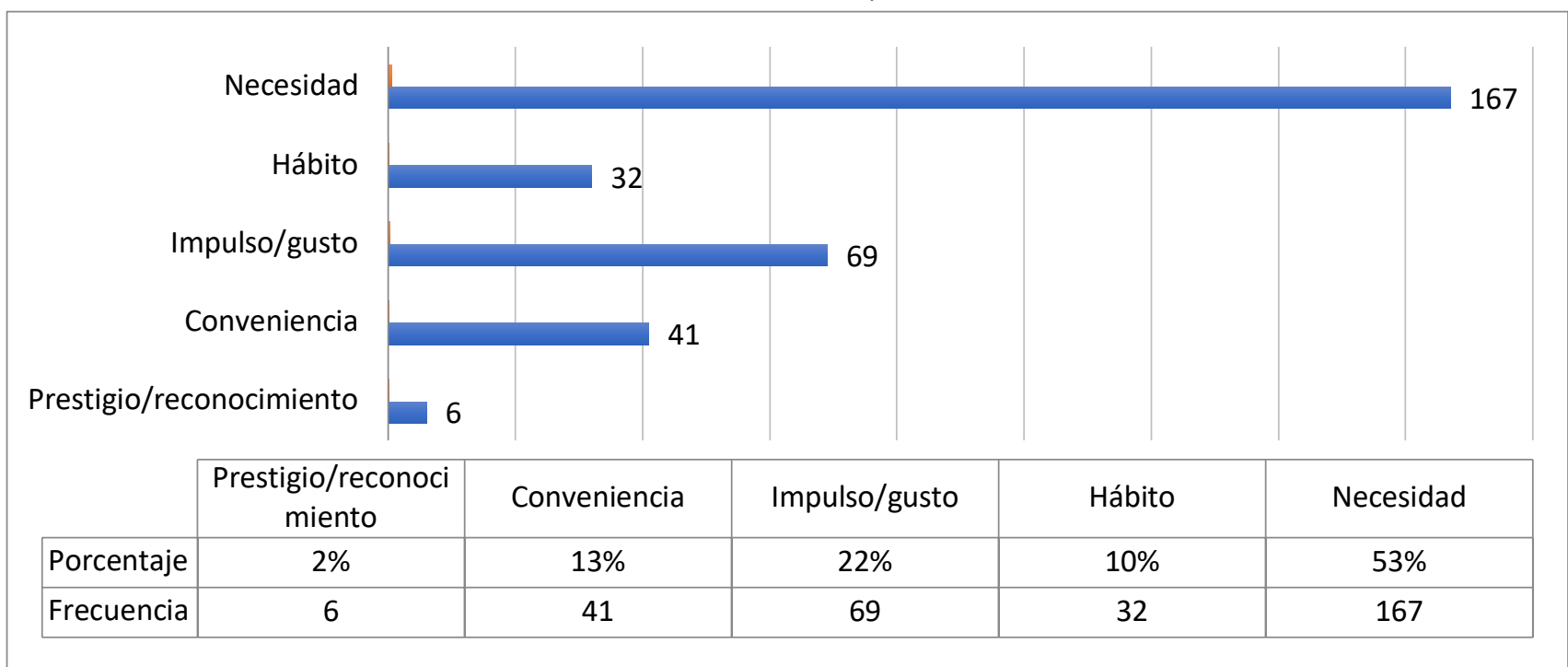

Fuente: Elaboración propia de los autores (2021)

Entre los canales de comunicación para venta implementados por las empresas del sector de restaurantes en la provincia de El Oro, para la compra de servicios de delivery se evidencia preferencia por las redes sociales (48\%) y las Ilamadas telefónicas (33\%). Se identifica que las aplicaciones móviles (16\%) son un canal en crecimiento (Ver gráfico 11).

Gráfico 11

Canales de comunicación para venta

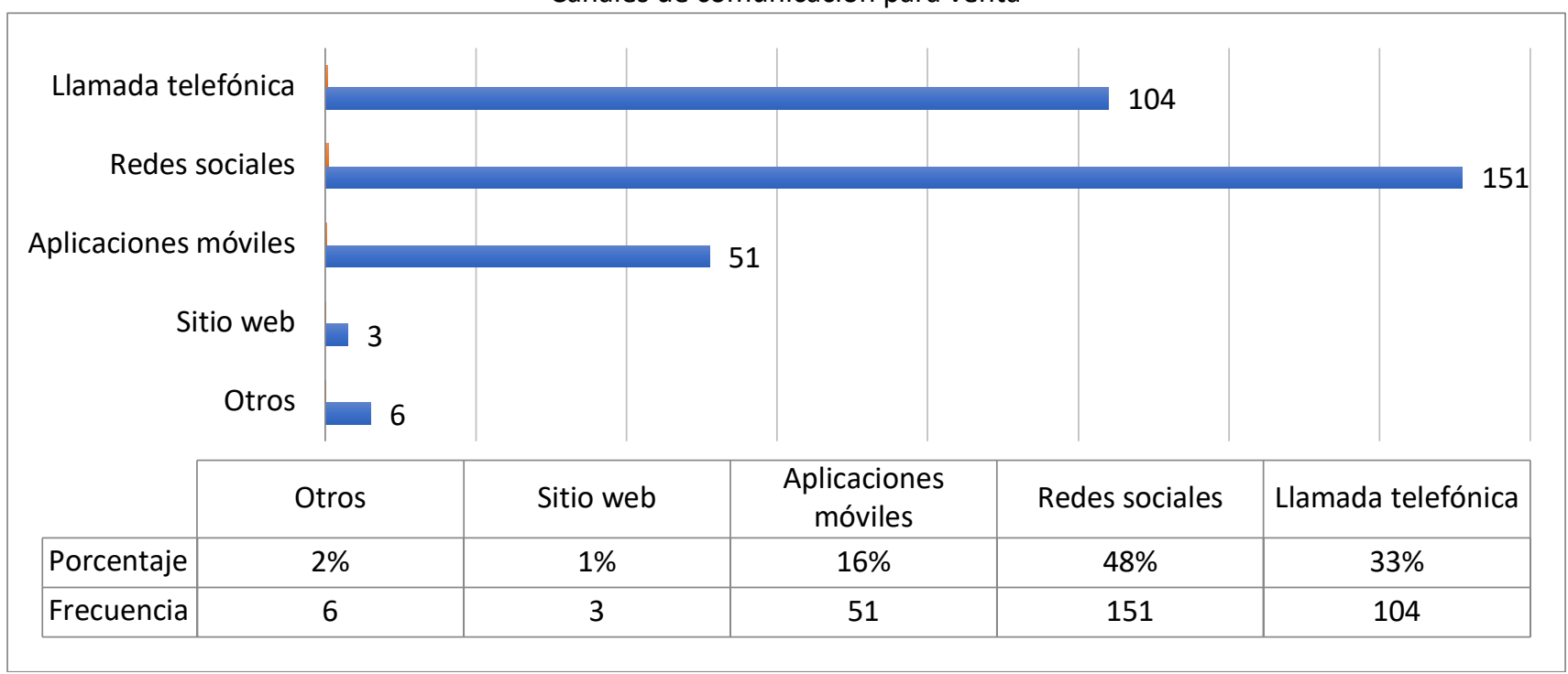

Fuente: Elaboración propia de los autores (2021)

\subsection{Discusión}

El contexto de desarrollo la investigación es la pandemia, habiendo un antes y después en el comportamiento del consumidor millennial frente al consumo de los servicios de restaurantes ante la llegada de la Covid-19. Este segmento prefería salir a comer, beber y socializar para vivir la experiencia, visitar el lugar era un motivo de 
distracción y entretenimiento con amigos y/o familiares; no obstante, el periodo de confinamiento trajo consigo un escenario de crisis que se convirtió en una oportunidad para las empresas del sector de restaurantes debido al incremento de la demanda del servicio de entrega a domicilio, esto como una respuesta del mercado ante la necesidad de consumo, protección y seguridad personal.

La investigación evidencia que los nacidos en los últimos años de la generación millennial (1996 al 2000) son los que, por necesidad o por adaptación, cambiaron su comportamiento habitual de consumo asociado al sector de restaurantes. El perfil de este segmento corresponde a mujeres solteras que actualmente tienen entre 21 y 25 años de edad y que estudian al tiempo que trabajan, por lo que manejan un nivel ingresos entre $\$ 200$ y $\$ 600$ dólares. En relación a sus necesidades, su prioridad es el consumo de comida rápida y parrilladas haciendo uso del servicio de delivery, esperando que la entrega en la comodidad de su hogar se realice de forma rápida, segura y dando cumplimiento a los protocolos de bioseguridad para garantizar salud e higiene; siendo oportuno destacar que la calidad sigue siendo importante pero no es la prioridad número uno en el contexto actual. Además, la puntualidad y el trato amable y respetuoso son factores que valoran e influyen en su percepción de marca y elección de compra.

En relación a indicadores específicos del comportamiento del consumidor, se identificó como lugares de compra con servicio de delivery a Chesco Pizzería y KFC. La frecuencia de consumo priotaria es mensual y habitualmente semanal, siendo el sábado el día de mayor demanda durante el fin de semana. En esta generación la decisión de compra surge por la necesidad de consumo, sin embargo, la demanda del servcio se da también por impulsoo o gusto; en este indicador el millennial se ve influenciado por el precio y la publicidad. El canal de comunicación habitual utilizado para contratar el servicio de delivery es la llamada telefónica, no obstante, los resultados demuestran que las redes sociales son en la actualidad el canal de preferencia para informarse de la oferta y demandar el servicio de entrega a domicilio. Cabe destacar que la utilización de aplicaciones móviles para realizar los pedidos de delivery evidencia un rápido crecimiento.

Finalmente se comprueba que la demanda de cómida rapida se mantiene a pesar de la crisis sanitaria, mientras que, el consumo de parrilladas ha incrementado considerando que la preferencia del consumidor en relación a este tipo de comida era vivir la experiencia de consumirla en el punto de venta físico. Los conusmidores se han adaptado a nuevas modalidades de consumo y nuevos canales de venta, los millennials son parte de la era digital y por ello se ven atraídos y se adaptan con facilidad a las innovaciones que se ofrecen en el mercado.

\section{Conclusiones}

Se concluye que la crisis sanitaria por la llegada del COVID-19 trajo consigo importantes cambios en el consumo y comportamiento del mercado del sector de restaurantes, como ha sido en el casco comercial de la Provincia de El Oro, donde tanto empresas como consumidores se han adapatado. Las empresas de este sector deben cambiar el enfoque en cuanto a la distribución, deben trasladar la experiencia de consumo a la intimidad del hogar, por ende resulta necesario implementar la entrega a domicilio como una estrategia clave y complementaria al consumo en el propio establecimiento, además de habilitar nuevos canales de comunicación para venta online $y$, de mantener los protocolos de bioseguridad. Los consumidores millennials son clientes informados que priorizan su necesidad y la seguridad del servicio de entrega a domicilio antes que la notoriedad de la marca; por necesidad o por adaptación, buscan experiencias de consumo que los satisfagan desde casa demandando rápidez, seguridad, salud e higiene en el contexto actual. Para los millennials una comunicación directa online con las marcas del sector de restaurantes se vuelve primordial por el acceso a información rápida y variada que pueden encontrar, considerando sus habilidades para el consumo de contenidos y el uso de las nuevas tecnologías. 


\section{Referencias bibliográficas}

Torres, V. (2013). La importancia del Subsector Hoteles, Bares y Restaurantes en la economía ecuatoriana durante la última década. Pontificia Universidad Católica del Ecuador. Obtenido de http://repositorio. puce.edu.ec/bitstream/handle/22000/6863/7.36.001446. pdf?sequence=4\&isAllowed=y

UNIVERSO, E. (2 de Abril de 2021). Las apps de entrega de comida a domicilio que podemos usar en Ecuador. EL UNIVERSO. Obtenido de https://www.eluniverso.com/noticias/ecuador/las-apps-de-entrega-de-comida-adomicilio-que-podemos-usar-en-ecuador-nota/

Casco, A. R. (2020). Efectos de la pandemia de COVID-19 en el comportamiento del. INNOVARE Revista de Ciencia y Tecnología.

COMERCIO, E. (05 de Mayo de 2021). El confinamiento impulsa otra vez al 'delivery' en Ecuador. EL COMERCIO.

Tene, E. (2020). Los impactos en la econom'ía del Ecuadorocasionados por el Coronavirus. Research Gate, 1-3.

Ramírez, D., Otero, M., \& Wilson, P. (2014). Comportamiento del. Criterio Libre, 165-183.

Reynolds Mejía, L. A. (2013). Percepciones y preferencias del consumidor de palmito fresco. Caso: unión de asociaciones de productores de plantines y palmito. PERSPECTIVAS, 61-104.

Ordaz, D., \& Osorio, M. (2018). Significados del viaje turístico en jóvenes millenials. Caso de estudio: Texcoco, México. Revista Latinoamericana de Ciencias Sociales, Niñez y Juventud, , 897-912.

RANDHAWA, R. S., BIRARI, A., \& JOSHI, A. (2014). Optimization of home delivery process using shortest path and fixed time method to reduce cost and investment. [EbscoHost]., 43-65.

Marquez, L. (2015). Optimización de rutas de reparto mediante la utilización de un modelo matemático optimizado por algoritmos genéticos. 1-15.

Medina, F., Román, Q., \& Patrón, R. (2020). Marketing proactivo como estrategia en Mipymes del sector restaurantero para afrontar Mercados encrisis por la pandemia covid-19. Revista Electrónica sobre Cuerpos Académicos y Grupos de Investigación, VII. Obtenido de http://www.cagi.org.mx/index.php/CAGI/article/view/216/428

Pérez, M. V. (2018). Marketing sensorial para el proceso de decisión de compra en el cliente del sector floristería. MARKETING VISIONARIO. Obtenido de https://dialnet.unirioja.es/descarga/articulo/7113498.pdf

Avila, B. (27 de Mayo de 2021). Ecovis Ecuador. Obtenido de https://ecovis.com.ec/impacto-del-covid-en-laeconomia-ecuatoriana/

Otzen, T., \& Manterola, C. (2017). Técnicas de Muestreo sobre una Población a Estudio. Int. J. Morphol, 227232. Obtenido de https://scielo.conicyt.cl/pdf/ijmorphol/v35n1/art37.pdf

Hernández, C. E., \& Carpio, N. (2019). Introducción a los tipos de muestreo. Revista ALERTA, II(1). doi:https://doi.org/10.5377/alerta.v2i1.7535 
Jordán, J., Ballesteros, L., Guerrero, C., \& Pérez, C. (2018). Determinantes en el comportamiento del consumidor que influyen en las decisiones gerenciales publicitarias en el sector comercial de la Provincia de Tungurahua. Revista Científica Dominio de las Ciencias, IV(4), 98-113.

Santos, I. (2019). El comportamiento del consumidor y las nuevas tendencias de consumo ante las TIC. Esic Market Economics and Business Journal, 621- 642.

Schiffman, L. (2010). Comportamiento del consumidor. México: PEARSON EDUCACIÓN.

Martínez, G., \& López, M. (2016). La influencia de los blogs de moda en el comportamiento del consumidor: Un enfoque exploratorio. Revista de Comunicación Vivat Academia, 85 - 109.

Quezada, M., Gualán, S., \& Ávila, V. (2020). Análisis del comportamiento de compra del segmento millennials frente a la pandemia Covid-19. Digital Publisher, 300-313.

\section{(cc) $\mathrm{EY}-\mathrm{NC}$}

Esta obra está bajo una Licencia Creative Commons Atribución-NoComercial 4.0 Internacional 